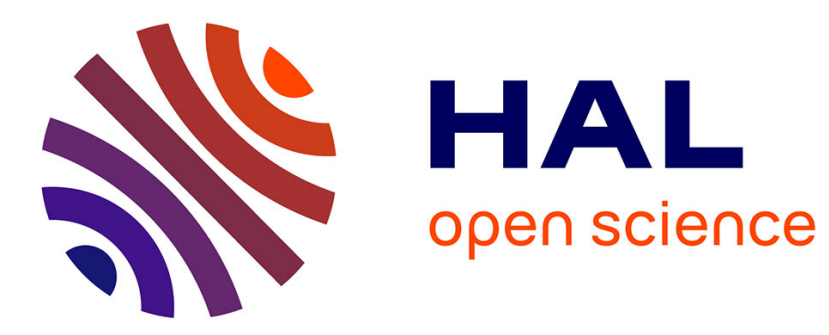

\title{
Propagation of acoustic waves in a one-dimensional array of noncohesive cylinders
}

\author{
Jean Rajchenbach, Xavier Noblin, Guillaume Huillard
}

\section{To cite this version:}

Jean Rajchenbach, Xavier Noblin, Guillaume Huillard. Propagation of acoustic waves in a onedimensional array of noncohesive cylinders. Physical Review E : Statistical, Nonlinear, and Soft Matter Physics, 2011, 84, pp.016602. 10.1103/PhysRevE.84.016602 . hal-00625668

\section{HAL Id: hal-00625668 https://hal.science/hal-00625668}

Submitted on 22 Sep 2011

HAL is a multi-disciplinary open access archive for the deposit and dissemination of scientific research documents, whether they are published or not. The documents may come from teaching and research institutions in France or abroad, or from public or private research centers.
L'archive ouverte pluridisciplinaire HAL, est destinée au dépôt et à la diffusion de documents scientifiques de niveau recherche, publiés ou non, émanant des établissements d'enseignement et de recherche français ou étrangers, des laboratoires publics ou privés. 


\title{
Propagation of acoustic waves in a one-dimensional array of noncohesive cylinders
}

\author{
Guillaume Huillard, Xavier Noblin, and Jean Rajchenbach \\ Laboratoire de Physique de la Matière Condensée, UMR 6622, CNRS, \\ Université de Nice Sophia- Antipolis, Parc Valrose 06108 Nice Cedex 2, France.
}

\begin{abstract}
By means of a photoelastic method, we access the visualization of acoustic waves propagating in a one dimensional array of noncohesive cylinders. As pointed by Nesterenko in the case of spherical grains (V.F. Nesterenko, J. Appl. Mech. Tech. Phys. 24, p. 567 (1983)), the nonlinearity of the contact law between the grains induces a dependence of the wave velocity both on its amplitude and on the confinement force. Our experimental method allows one to access the evolution in time of the internal state of stress of individual grains with an excellent accuracy. We show that the velocity of the sound presents two regimes as a function of the confining force. For low forces, the dependence is strongly nonlinear, while it weakens for higher forces. By means of the direct visualization of the contact zone, we show that both micro- and macroscale imperfections of the surface of contact explain the low forces behavior. We test the consistency of our experimental findings results with both the theoretical expectations and with the experimental determination of the force-displacement dependence. We show moreover that the main damping process originates in solid friction.
\end{abstract}

PACS numbers: 43.25.+y, 45.70.-n, 43.25.Ed, 46.40.Cd

Published in Phys. Rev. E 84, 016602 (2011)

\section{INTRODUCTION}

The disorder in the grain positions, the noncohesiveness of the material, the nonlinearity of the contact law between grains and the interferences between waves passing through different paths render arduous the theoretical treatment of the sound propagation in granular media [1-7] Nevertheless, understanding the properties of propagation of sound waves in granular media is of crucial interest, e.g., in order to design new acoustic attenuators or, for instance, to improve the interpretation of threedimensional (3D) acoustic tomographic data or seismic recordings.

Since waves are transmitted from one grain to some of its neighbors via the contact network, the nature of the contact plays a crucial role. Due to the nonlinearity of the contact law [8], the sound velocity depends on the initial state of the (static) pre-stress of each contact. Hence, both the force-displacement relationship between grains in contact and the topography of the contact network have to be accounted for. The second issue then proceeds from the packing disorder. Even if the positions of the grain centroids are ordered in a lattice, some contacts can be open owing to a very small deviation in the particle size or shape and the actual contact network is thus disordered [9]. It is well known that an applied external load induces a filamentary network of stress (called force chains) in granular packings, where a modest fraction of the total number of grains carries the main part of the force [10]. Note moreover that the passing of acoustic waves of large amplitude can in turn modify the contact topography. Interestingly, data obtained by Liu and Nagel [2] emphasized the role of interference between acoustic waves following different paths. The complex acoustic signal (the $\operatorname{coda}$ ) obtained in $3 \mathrm{D}$ geometries was interpreted as the signature of a speckle phenomenology, which emphasized the high sensitivity to geometrical rearrangements induced by, e.g., small temperature drift or by the wave propagation itself.

Due to the increase of the contact area with the normal load, the relation between the normal component $F_{0}$ of the contact force and the displacement $\delta_{0}$ between the grain centroids is nonlinear. For spherical beads, this relationship is known as Hertz's law [8] and reads $F_{0} \propto \delta_{0}^{3 / 2}$. As a consequence, the wave velocity $V$ increases with the static confining force $F_{0}$. Nesterenko modeled the sound propagation in a one-dimensional nonlinear array $[1,11]$, and he showed that various types of sound waves can propagate through these media, according to the wave amplitude:

(i) Provided that the amplitude of the sound wave is very small compared to the static confining force $F_{0}$, linear acoustic waves can propagate. Theory predicts that the linear wave speed increases as $V \propto F_{0}^{\beta}$, with $\beta=1 / 6$ for spherical beads. Experiments have been carried out in 1D, 2D and 3D for different packing geometries of spherical beads $[4,12,13]$.

(ii) At higher amplitudes, when both nonlinearity and dispersion play a role, a cnoïdal wave or a KortewegdeVries type soliton can be encountered.

(iii) For excitation amplitudes even higher than the confining force $F_{0}$, a new type of excitation (the Nesterenko's solitary wave) is predicted [1]. The speed of this solitary wave varies as the power $1 / 6$ of its amplitude. The existence of such a nonlinear excitations has been recognized experimentally and numerically $[1,3,14,15]$.

The present study is devoted to the propagation of a linear compressive pulse along a 1D array of photoelastic cylinders. Instead of measuring the flight time of the pulse through the array, here we have direct access both to spatial and temporal quantitative informations by means of a photoelastic visualization, which is in real time. The high photoelastic constant of the material 
allows for an accurate determination of the stress state of each individual grain as a function of time, with a sampling rate up to $10^{+5} \mathrm{~s}^{-1}$. Shukla et al. were the first to use the photoelastic method to probe dynamically wave phenomena, mostly in the non linear regime $[16,18]$. In a previous paper [19], we have presented a simple quantitative way to measure forces using the photoelastic technique. We focus here on the linear waves speed, and their dissipation.

One of the goals of the present work is to highlight the mechanism which governs the dependence of the wave speed on the imposed static confining force. In some cases, using various materials (steel, brass, glass and nylon spheres), the Hertzian prediction with $\beta=1 / 6$ has been recovered in 1D arrays [12]. Nevertheless, deviations from the above expected value has been reported for low confining forces, with experimental determinations of $\beta$ giving a value close to $1 / 4$, that both in $1 \mathrm{D}$ [20], 2D [13], and 3D geometry [4, 21].

Two reasons have been invoked to explain this discrepancy. The first one relies on the disorder of the packing: some contacts, initially open, are progressively closed when the static confining pressure is increased. Therefore, the macroscopic compressibility of the sample is different at low or high pressure [22]. Another explanation involve a local deviation to sphericity at a microscopic level [23]. The discrimination between these two different mechanisms has not been elucidated yet. To sum up, the relation between the experimental value of $\beta$ and the nonlinearity in the contact law remained unclear.

In the present paper we show that the speed increases as a power $\beta \approx 1 / 4$ of the confining force $F_{0}$, for the low values of the latter. For larger values of $F_{0}$, the exponent $\beta$ is significantly reduced. Our experiments reveal that these deviations from the expected velocity dependence on $F_{0}$ originate in both micro and macroscale imperfections of the contact and subsequent unexpected variations of the surface contact areas with pressure.

Another aim of this work is to study the attenuation of the pulse amplitude while propagating along the array, and to propose a simple model accounting for it.

In the following, we describe first the photoelastic method and the experimental setup (Sec. II A and II B). We then present then experimental results concerning the wave velocity as a function of the static confining force (Sec. III A), the related variation of the mean contact area (Sec. III C) and the force-displacement law (Sec. III B). Next, results concerning the damping processes are shown (Sec. IIID). In the last section (Sec. IV), we discuss and propose some elementary explanations capable of accounting for our experimental findings.

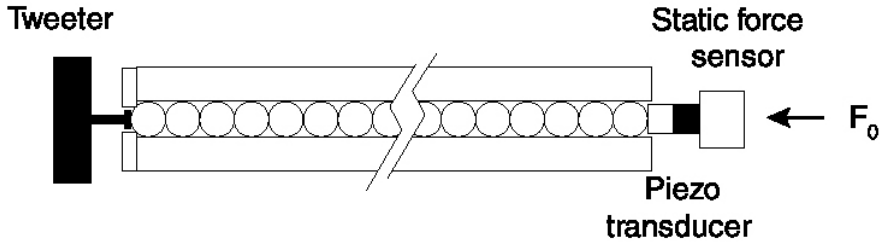

FIG. 1: Experimental setup showing 1D grain chains

\section{EXPERIMENTAL SETUP AND METHOD}

\section{A. Setup}

We use a photoelastic method to obtain quantitative information on the stress state of individual grains in a $1 \mathrm{D}$ array. The experimental setup is sketched in Fig. 1. The system that we studied consists of a linear array of 36 identical cylinders. Grains are prepared by cutting a 9.4 mm-thick polycarbonate plate and machining identical cylinders with diameter $d=2 R=13 \mathrm{~mm}$. The Young's modulus, the Poisson ratio and the mass per unit of volume of polycarbonate are respectively $E=2.76 \mathrm{GPa}$, $\nu=0.38$ and $\rho=1,2 \mathrm{~g} . \mathrm{cm}^{-3}$. Most of the previous studies dealt with spherical grains, while here we use cylinders. Note moreover that the topological disorder proper to $2 \mathrm{D}$ or $3 \mathrm{D}$ geometries does not intervene in the $1 \mathrm{D}$ geometry that we probe here, so that, the acoustic behavior originates merely on the nature of the contacts.

The elastic grains are then packed into contact along a common generatrix, and confined in a $470 \mathrm{~mm}$ long duraluminium frame. To ensure the alignment of the cylinders in the parallel direction of their axis, a groove (0.2 $\mathrm{mm}$ deep and $10 \mathrm{~mm}$ wide) has been machined along the bottom plate of the cell. Moreover, grains can simultaneously undergo an horizontal confining force $F_{0}$ and a vertical force $F_{\perp}$. This last one is applied by loading the top lid and prevents the natural buckling of the chain. Both these forces can be varied and measured with precision.

A mechanical pulse generated by means of a tweeter impacts the first grain of the array. A piezoelectric sensor and a static force sensor are positioned after the last grain of the array. They allow access to the static confining force $F_{0}$ and the pulse amplitude. They are complementary to direct photoelastic measurements performed in situ. In order to increase the resolution relative to the position of the birefringence fringes, the light is filtered by means of an interferential filter centered around 650 $\mathrm{nm}$. The propagation of the acoustic pulse through the array is then recorded by means of a high speed digital camera (Phantom 7.3) with a resolution of $736 * 32$ pixels. Note that a cylinder diameter corresponds typically to 30 pixels. The sequence of images is then processed and analyzed by means of a program written in Matlab language. The bases of the photoelastic method are discussed below. 


\section{B. The photoelastic method}

Consider a photoelastic cylinder positioned between a couple of identical circular polarizers. Light propagates through grains along the direction parallel to their axis of symmetry. The emerging light intensity is a function of the stress in the plane of the cylinder at each point $(x, y)$ and reads as:

$$
I(x, y)=I_{0}(x, y) \cos ^{2}\left[\frac{2 \pi}{\lambda} C e\left[\sigma_{1}(x, y)-\sigma_{2}(x, y)\right]\right]
$$

where $I_{0}$ is the incident intensity, $\lambda$ is the wavelength of the light, $C$ is the photoelastic constant of the sample, $e$ is the thickness of the sample and $\sigma_{1}, \sigma_{2}$ are the local principal stresses at the point $(x, y)$. This relation holds everywhere in the material.

In order to access the state of stress of the individual grains, we compare the light intensity in the central region of each grain with that obtained by means of a calibration procedure. This calibration consists of measuring the transmitted light as a function of the amplitude of an adjustable external uniaxial load $F$. The central region of measurement is less than $1 \%$ of the surface of a grain. The calibration curve is shown in Fig. 2. The intensity measured at the center of the grain, $I_{\text {center }}$, can be expressed as:

$$
I_{\text {center }}=\left(I_{\max }-I_{\min }\right) \cos ^{2}\left[\pi \frac{F}{\Delta F}\right]+I_{\min }
$$

where $F$ is the compressive force, and $\Delta F$ is the force increment corresponding to the passing of a black fringe to the following black fringe. $I_{\max }$ is the maximum transmitted intensity and $I_{m i n}$ is the residual illumination corresponding to the black fringe. In the following, we note:

$$
\zeta=\frac{\left(I_{\text {center }}-I_{\min }\right)}{\left(I_{\max }-I_{\min }\right)}
$$

$\zeta$ is the normalized intensity transmitted at the center of the cylinder and takes value between 1 (white fringe) and 0 (black fringe). We can therefore access to the force $F$ by computing:

$$
F=\Delta F\left[(n-1)+\frac{(-1)^{n-1}}{\pi} \arccos (\sqrt{\zeta})\right]
$$

where $n$ is the fringe order. We measure for our grains $\Delta F=42 \mathrm{~N}$. Note that this method is only applicable for forces of the same order on both sides of a grain, with the resulting measurement giving their average value.
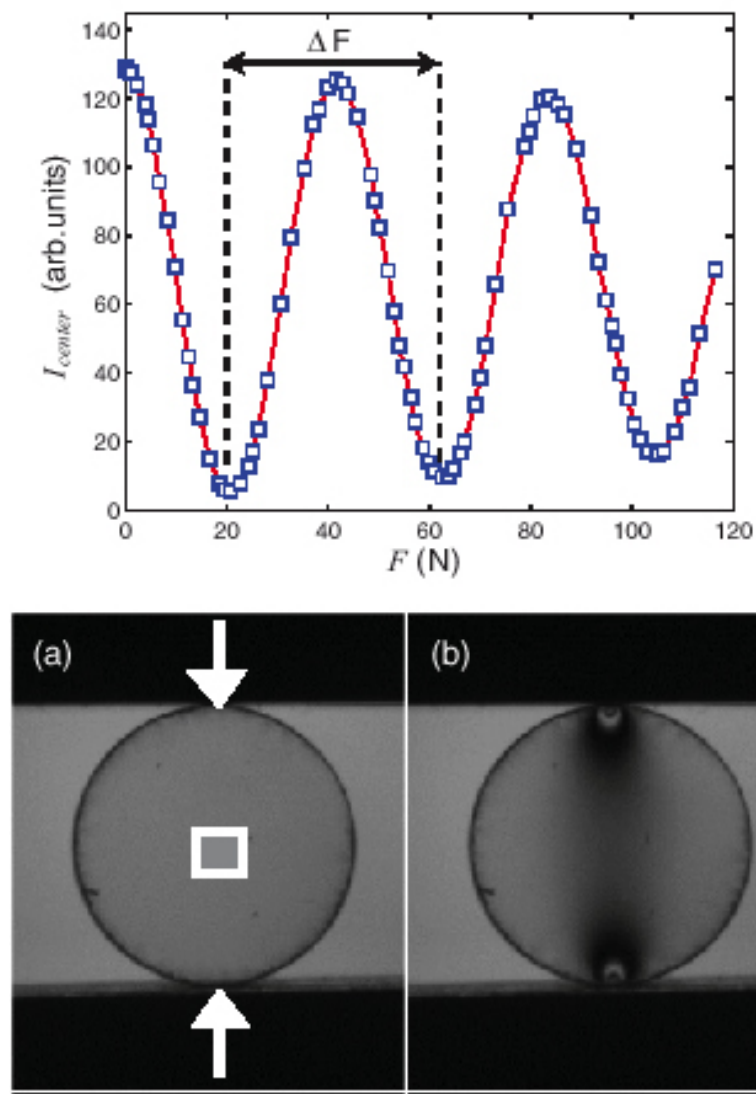

(b)

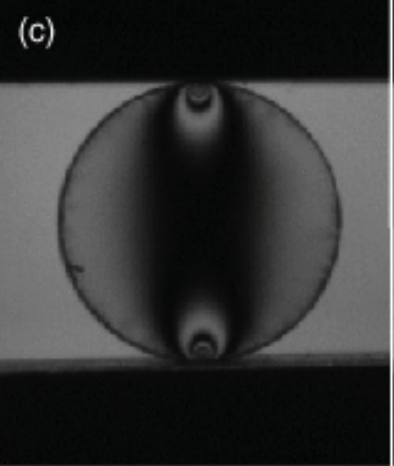

(d)

FIG. 2: (Color online) Photoelastic calibration: we measure the intensity $I_{\text {center }}$ transmitted through the central region of one grain (white box in (a)) as a function of an applied external uniaxial force $F$. We deduce from it the force increment $\Delta F$ corresponding to the passing of a black fringe to the following black fringe. For cylinders with $13 \mathrm{~mm}$ diameter, we measure $\Delta F=42 N$. (a)-(d): Photoelastic images obtained with the calibration for forces $F$ equal to $0,10,20,40 \mathrm{~N}$.

\section{Procedures}

The typical protocol used to conduct measurements is the following. First, particles are positioned in the groove. For a measurement corresponding to a given static confining force $F_{0}$, the vertical force $F_{\perp}$ is adjusted in order to ensure the vertical alignment of the array. Then the closest static forces corresponding to black and 


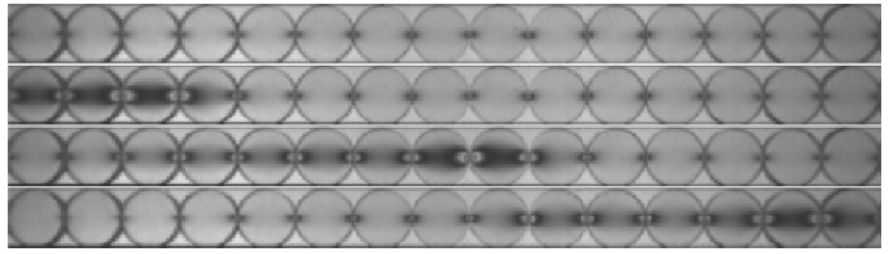

FIG. 3: Example of four successive photoelastic images showing the acoustic pulse propagation. The time between each images is $140 \mu s$ and the static confining force is $F_{0}=4.5 \mathrm{~N}$. The maximum amplitude of the acoustic pulse is $f_{1, \max }=$ $10 N$ and the pulse durationis $100 \mu \mathrm{s}$. Before the pulse is sent (top image), grains are subjected to the static force $F_{0}$, which causes the slight fringes near each contact. Then, compressive pulse arises and travels in the chain with a velocity $V$.

white fringes located in the grain centers are measured. Hence the values of $I_{\max }$ and of $I_{\min }$ are deduced for each particles. Note that the determination of $I_{\max }$ and $I_{\min }$ are required to measure the transient force corresponding to the passing of the pulse. Then a function synthesizer generates a pulse to the tweeter (time-width $100 \mu s$ ) and a sequence of pictures is recorded with a frame rate $89000 \mathrm{fps}$. After the tweeter has impacted the first grain, an acoustic pulse propagates through the chain. A sequence of pictures showing the propagation of the pulse is showed in Fig. 3. Note that the spatial extension of the wave is limited to a few grains. Our image analysis program allows one to access the quantity $\zeta$ and then the dynamical force $F_{n}(t)=F_{0}+f_{n}(t)$ for each step of time and for each particle (where the index $n$ is relative to the grain position in the image. In most cases, the first grain of the image corresponds to the third grain of the chain). In the following, the maximal amplitude of the pulse for the grain $n$ is noted $f_{n, \max }$, i.e.:

$$
f_{n, \max }=\max \left[F_{n}(t)-F_{0}\right]
$$

\section{EXPERIMENTAL RESULTS}

\section{A. Wave velocity in the linear regime}

Fig. 4 shows the different curves of $F_{n}(t)$ for the grains numbered $n=8,13,18,23$ as a function of the time. Before the pulse emission, all the curves coincide and correspond to the static force $F_{0}$. The measurement of $F_{0}$ proceeds from two independent determinations: first, $F_{0}$ is measured by means of the photoelastic method, but, we can also access the data issued from the static force sensor. Nevertheless, note that a discrepancy can arise, which originates in the solid friction with the confining boundary walls. As a consequence, when this effect is large, the photoelastic method is preferred. Then, for a given grain, the pulse arises and the force reaches the maximal value before decreasing and oscillating. Note
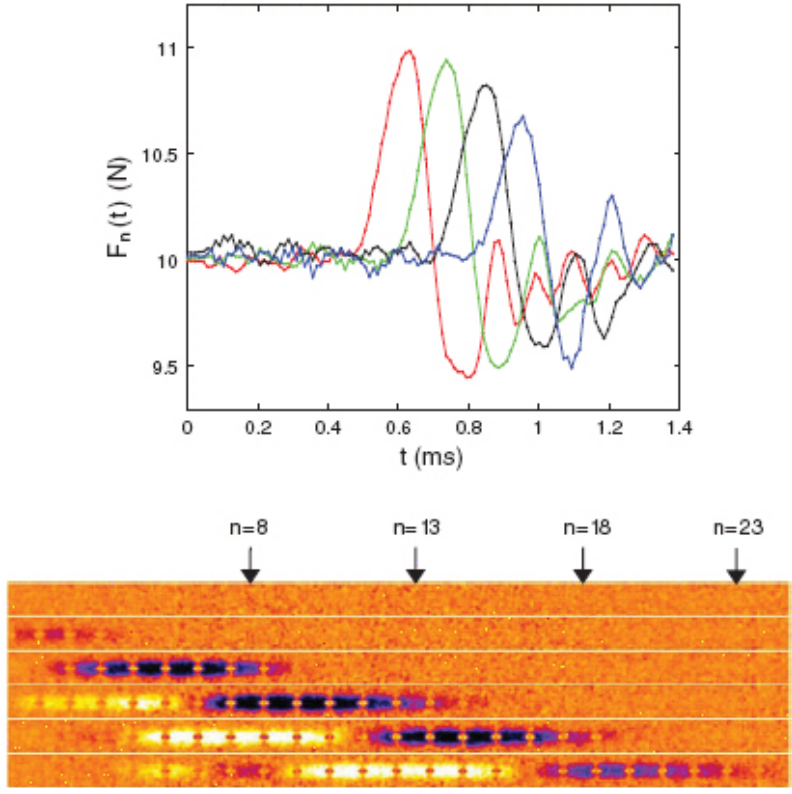

FIG. 4: (Color online) Top: Dynamical force $F_{n}(t)$ vs time for grains $n=8,13,18,23$ (from the left to the right) in the linear regime. These curves are the result of the data analysis of photoelastic images by computing Eq. 4 for each grain. Before the pulse arises $\left(f_{1, \max }=1.3 \mathrm{~N}\right.$ for the first grain), each grain is submitted to the static force $F_{0}=10 \mathrm{~N}$, then the pulse travels from one grain to another. The temporal width is quite constant for each grain but the maximal amplitude $f_{n, \max }$ decreases. Bottom: image sequence from which the curves have been determined. The grain used have been marked, we have divided the image by the first one of the movie, and used false colors.

that in this set of experiments, $f_{1, \max }$ is always very small compared to $F_{0}$ which ensures the regime to be linear. We observe that the wave propagates through the array, and that the spatial extent of the pulse remains constant during its propagation (within our experimental precision). The spatial width of the pulse is typically $6-7$ grain sizes, so that dispersive effects intrinsic to discrete lattices are negligible along the present chain comprising 36 cylinders. Considering an underestimated wavelength of seven grain sizes, we find that the speed difference between the linear dispersion relation (Eq. 11) and the actual sinusoidal one for the spring-mass chain is about $3 \%$, which is small. Interestingly, we note that the compressive pulse is followed by a tensile component (the detailed analysis of this phenomenon is beyond the scope of this paper). Finally we note that the amplitude of the pulse decreases uniformly along the chain. The origin of this attenuation will be discussed in-depth in Sec. III D.

From the measurement of the time corresponding to the mid-height pulse amplitude located at the grain of index $n$, we deduce the distance covered by the pulse as a function of the time (see Fig. 5). This dependence is fairly linear, whatever the confining force. We can de- 


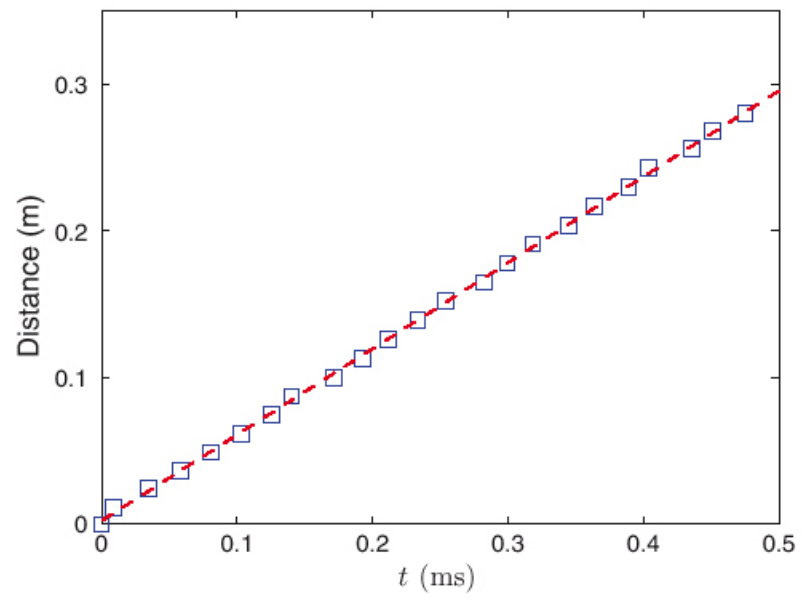

FIG. 5: (Color online) Distance covered as a function of the time for the wave mid-height of the Fig. 4. The dashed line is a linear fit giving the velocity $V$.

duce therefore the celerity of the wave as a function of $F_{0}$ (see Fig. 6). Clearly, two regimes have to be distinguished. For $F_{0}<F_{c} \approx 20 N$ the wave speed increases rapidly with the static force $F_{0}$. Assuming a power law dependence for the velocity as $V \propto F_{0}^{\beta}$, we determine an exponent $\beta \approx 1 / 4$. This is a clear signature of a highly nonlinear contact law. On the other hand, Fig. 6 shows a significant decrease of the exponent $\beta(\beta \approx 1 / 9)$ for a larger confining force $\left(F_{0}>F_{c} \approx 20 N\right)$. Actually this regime seems more consistent with the theoretical prediction suited for cylinders, for which the displacementforce relationship is logarithmic (see Sec. IV). In Fig. 6 , we have also traced the velocity-confining force curve $V^{\star}\left(F_{0}\right)$ calculated from $V^{\star}=\lambda V$ with $V$ given by Eq. 11 and $k\left(F_{0}\right)$ computed from the experimental static $F_{0}-\delta$ relation (see Fig. 8). We find good agreement and we interpret the two regimes as a consequence of a change in the contact law for increasing force due to roughness of the contact at microscales and discrepancy with the perfect cylindrical shape at macroscales. Nevertheless, a numerical coefficient has to be used, with $\lambda=1.48$ instead of 1 . This discrepancy may be due to the fact that in our frequency range the dynamical stiffness is higher than the static one.

To check the importance of the microscale roughness effect, we have also performed measurement with polished grains (see triangles, Fig. 7). Their microscopic roughness (arithmetic mean value) is about $R_{a} \simeq$ $0.37 \mu \mathrm{m}$ as compared to the non-polished grains for which $R_{a} \simeq 0.75 \mu \mathrm{m}$, the maximum crest to crest distance being respectively $2 \mu \mathrm{m}$ and $4 \mu \mathrm{m}$. The velocity curve presents roughly the same aspect as for unpolished grains, with a lower exponent at large forces. For low forces differences are not significant due to the relative poor precision on $F_{0}$. For high forces, local exponent have been measured as $\beta \simeq 1 / 9$ in the non-polished case and $\beta \simeq 1 / 11$ in the polished case. The difference in roughness was not

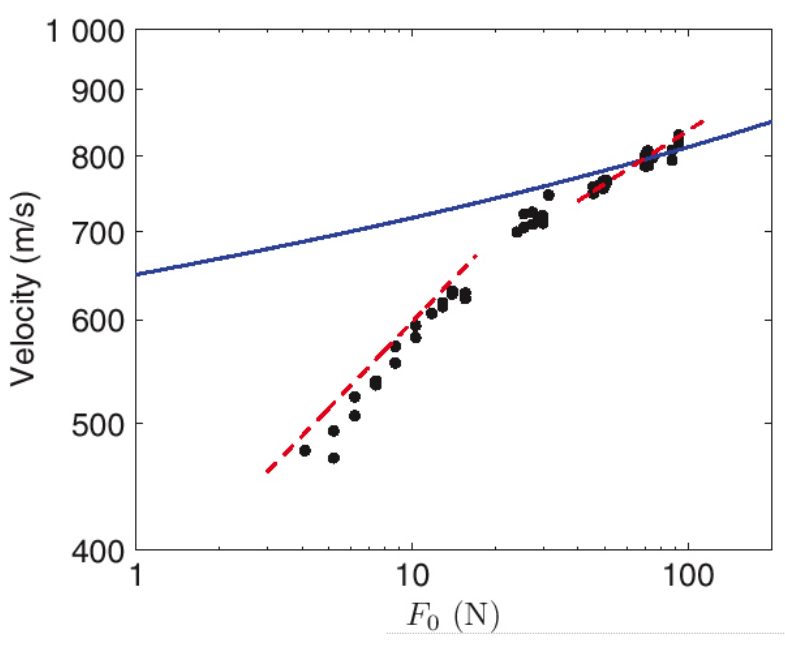

FIG. 6: (Color online) Plot, in log-log scale, of the wave velocity $V$ versus the static force $F_{0}$. For $F_{0}<F_{c} \approx 20 \mathrm{~N}$, velocity increases rapidly with the static force, we measure an exponent near to $1 / 4$. For $F_{0}>F_{c}$, the dependence of the speed wave with $F_{0}$ is weaker (exponent 1/9). The solid curve is the theoretical prediction based on Eqs. 10 and 11. The dashed lines are also deduced from Eq. 11 but using the stiffness determined from the experimental force-displacement law (see Fig. 8) and a numerical prefactor $\lambda=1.48$.

significant enough to find strong differences in the forcevelocity relationship, however at high forces a difference in the exponent could be highlighted, expressing the fact that the theoretical behavior for a perfect cylinder was recovered for these grains. The logarithmic contact law for cylinders gives locally an exponent close to $1 / 11$, which is in good agreement with the smooth surface grains that present behavior close to those of the perfect cylinders.

We have also performed measurements by cycling the variation of the confining force: $F_{0}$ was first increased and then decreased in order to detect the possible occurrence of hysteretic effects, originating in plastic events at contact. Actually, we did not observe any significant change of velocity up to $F_{0} \simeq 100 \mathrm{~N}$.

\section{B. Force-displacement relationship}

To access the static force-displacement relation of the array, we simply measured the total displacement of the chain for various $F_{0}$. Once again, precautions have to be taken in order to avoid chain buckling. Therefore, a vertical load is applied to the upper lid to prevent any buckling. Note that the vertical load must be moderate in order to avoid noticeable effects on solid friction. Indeed, noticeable friction between the horizontal grain array and the boundary walls would lead to an significant effective decrease of the confining force along the array (this phenomenon is discussed in depth in Sec. IIID. In Fig. 8, We have plotted the relation between the force $F_{0}$ and the displacement $\delta$ for one grain. One can 


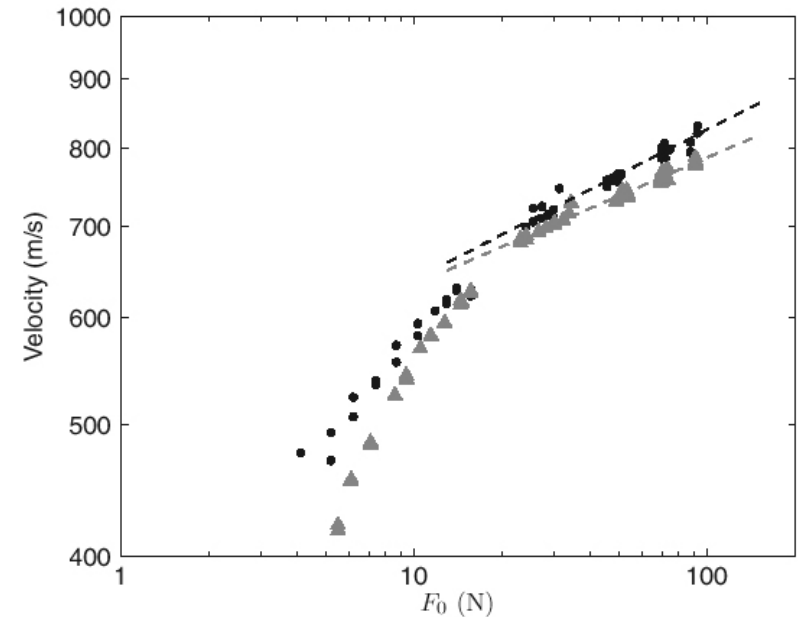

FIG. 7: (Color online) Plot, in log-log scale, of the wave velocity $V$ vs the static force $F_{0}$ for two surface roughness (triangle: smooth surface, circles: more rough surface). The behaviors at low forces are not representative due to larger errors on $F_{0}$ in this regime. For $F_{0}>F_{c} \approx 20 \mathrm{~N}$, the exponent $\beta\left(V \propto F_{0}^{\beta}\right)$ is equal to $1 / 9$ for the rough surfaces and $1 / 11$ for smoother surfaces. Straight lines are power fits in the high forces region.

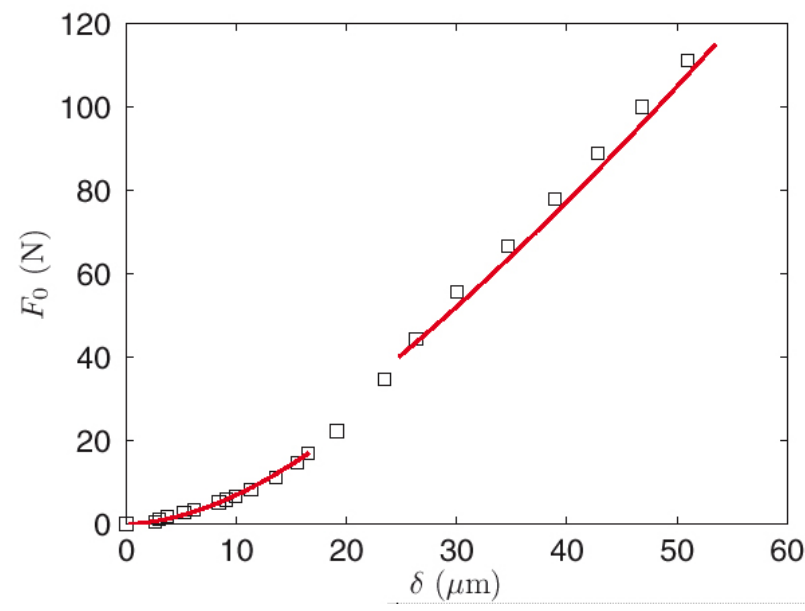

FIG. 8: (Color online) Static force-displacement relation between $F_{0}$ (in N) and $\delta$ in $(\mu \mathrm{m})$ for one contact between cylindrical grains. The solid curves are power fit $F_{0} \propto \delta^{\alpha}$ with $\alpha=1.78$ for small displacements and $\alpha=1.37$ at higher displacements.

clearly distinguish two different regimes, consistent with the $V-F_{0}$ curve. From these data, we can calculate an effective stiffness, and then using an elementary springmass model (see Section IV) it is then possible to predict the $V-F_{0}$ relationship in the studied system (see Fig. $6)$.

\section{Surface of contact between cylinders}

In order to have a better understanding of the crossover phenomenon exhibited by both the $V-F_{0}$ and $F_{0}-\delta$ dependences, and to the static force-displacement curve, we have performed a direct visualization of the contact zone between two adjacent cylinders. We access the variation of the contact areas as the static confinement load is increased by means of the following simple method. The array of grains is diagonally lightened up by means of a spotlight. The actual contact area appears as bright (because the light is scattered), while the unloaded regions of the cylinder surface appear as dark (see experimental setup in Fig. 9).

It is therefore straightforward to access the variation of the contact area as the external load is increased. In Fig. 9 a sequence of pictures shows the variation of the lightened actual contact between two adjacent grains as the static confining force is increased for two different families of grains (polished or non-polished). First we notice that a slight imperfection on the cylindrical shape of the grains leads to an incomplete contact along the cylinder generatrix. Second, as the force is increased, the microscopic roughness induces a local increase of the contact area. Using image analysis, we determine the variation of the contact area with $F_{0}$. Results are presented in the Fig. 10. Clearly, for the collection of the polished grains, the behavior expected for ideal cylinders is met as the confining force $F_{0}$ is larger than $\approx 20 \mathrm{~N}$. For the case of non-polished grains, the theoretical slope is recovered roughly above $50 \mathrm{~N}$. In both case, the cross over is indicated by an arrow in Fig. 10, it also corresponds to the velocity change as function of $F_{0}$ (see Fig. 6). From both micro- and macroscale defects of cylinder surfaces, it results, at low force, a more rapid increase of the contact areas with $F_{0}$, compared to the case of ideal cylinders in contact along a common generatrix.

\section{Dissipation}

In the following, we address the issue of the dissipation processes which governs the pulse damping during its propagation. Figures 4 and 11 clearly show a fairly linear decrease of the pulse amplitude with the covered distance. Interestingly, in Fig. 11, the decay slope does not appear to depend significantly on the initial pulse amplitude $f_{1, \max }$. Let us define $\alpha$ as the slope of the decay law of the pulse amplitude with distance (expressed in cylinder diameter unit). Hence $\alpha$ (defined in force unit) represents the decrement of the force amplitude when the pulse is transmitted from one grain to its following neighbor. Note that the slope $\alpha$ may depends on the lateral confining static force $F_{0}$ and on the vertical load $F_{\perp}$ applied on the top lid. These features will be discussed in Sec. IV.

An attenuation length $L_{a}$ (expressed here in grain di- 

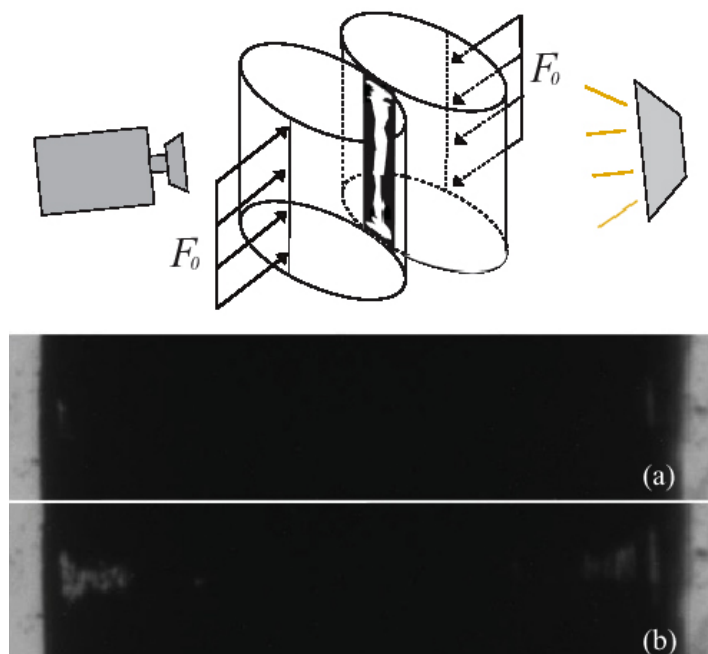

(b)

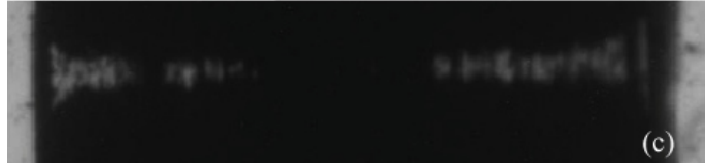

(c)

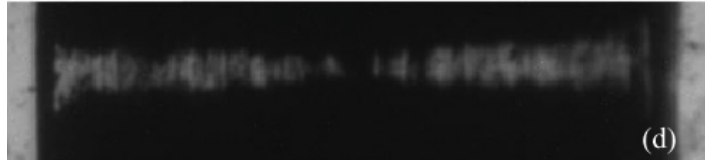

(d)

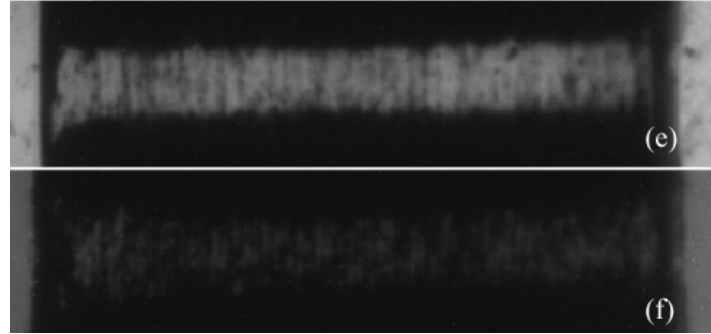

FIG. 9: (Color online) Top: Experimental setup to visualize the contact area. When grains are in contact, they deform themselves and the surface becomes locally plane. Thus, light is transmitted without reflection and pixels are illuminated. In the other case, because of the curvature of the surfaces, light is reflected and pixels appear darker. Bottom : Images of the contact between cylinders for different static forces $F_{0}$. (a)-(e) Contact surface between two polished cylinders for $F_{0}=0,3,9,22,90 N$. (f) Surface contact at $F_{0}=90 \mathrm{~N}$ for non-polished cylinders. We see a clear difference in comparison with (e).

ameter units) can be defined as the distance for which the pulse amplitude vanishes to zero. It is interesting to examine the dependence of the attenuation length $L_{a}$ both on lateral confining force $F_{0}$ and on the vertical load $F_{\perp}$ applied on the top lid.

In Fig. 12, the variation of the attenuation length $L_{a}$ is plotted as a function of the initial amplitude of the pulse, that for various confining forces $\left(F_{0}=8,14\right.$ or $26 \mathrm{~N})$ and vertical loads $\left(F_{\perp}=0.15,0.22\right.$ or $0.49 \mathrm{~N}$ per grain). In all cases, the attenuation length $L_{a}$ is seen to depend linearly on the initial pulse amplitude $f_{1, \max }$.

This behavior is consistent with Eq. 13. Note that the

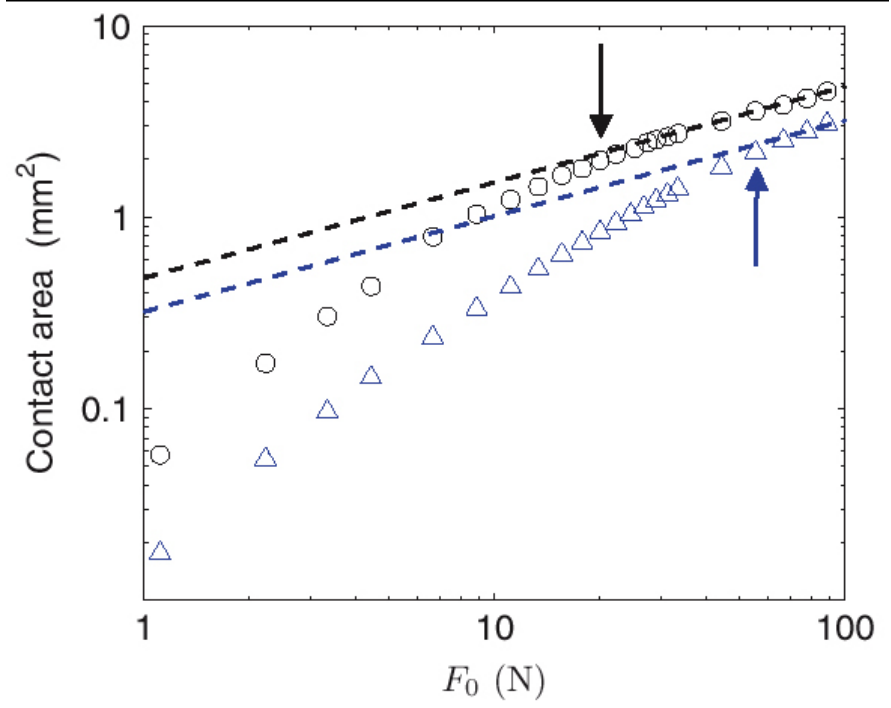

FIG. 10: (Color online) Contact area versus static force $F_{0}$ for polished cylinders $(\bigcirc)$ and non- polished cylinders $(\triangle)$. The dashed lines are a guide to the eyes, as power law $F_{0}^{1 / 2}$ which corresponds to the theoretical law for perfect, smooth cylinders (see Eq. 6). The two arrows indicate the cross over forces as discussed in the text.

experimental points $(\diamond$ and $\nabla$ ) obtained with the same vertical load $F_{\perp}\left(F_{\perp}=0.15 \mathrm{~N}\right.$ per grain $)$ and two different values of the lateral confining force $\left(F_{0}=8\right.$ and $14 \mathrm{~N})$ merge on the same line. That proves that the damping effect does not vary significantly with the lateral confining pressure $F_{0}$. On the other hand, the set of points displayed in Fig. 12 shows that the attenuation length decreases as the vertical load $F_{\perp}$ is increased. As a consequence, $\alpha$ only depends on the vertical force $F_{\perp}$.

Next, in Fig. 13, we plot the coefficient $\alpha$ as a function of $F_{\perp}$; it appears unambiguously that $\alpha$ increases linearly with $F_{\perp}$. This behavior is interpreted in the following section.

\section{MODEL AND DISCUSSION}

\section{A. Model}

Current models aimed at describing the propagation of acoustic waves through a linear array of grains rely on the analogy with a system of masses and springs [11]. For a system of spheres in contact, springs must be considered as nonlinear, to be consistent with Hertz's law, which expresses the relation $F_{0} \propto \delta^{3 / 2}$ between the compression force and the displacement $\delta$ between the sphere centroids. This last relation [8] proceeds from the exact equilibrium solution of the linear elasticity equations, and the nonlinearity of the Hertz law results merely from the increase of the area of contact with $F_{0}$. It is worth noting that the contact is unilateral, that is, the contact force becomes zero as soon as the distance 


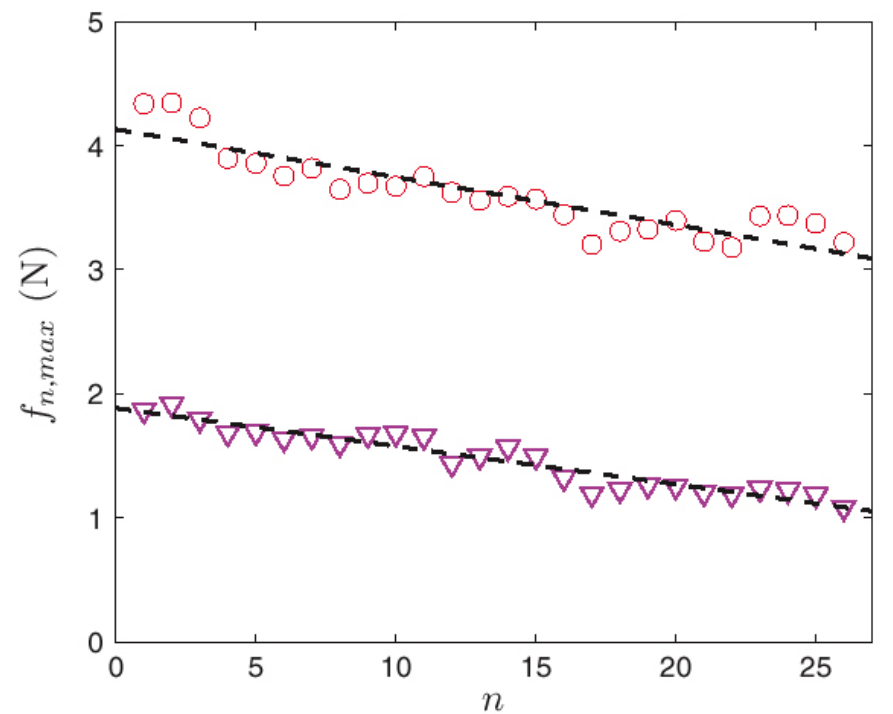

FIG. 11: (Color online) Plot of the maximum amplitude $f_{n, \max }$ vs grain number $n$. The two sets of data points correspond to different initial pulse amplitude: $f_{1, \max }=1.9 \mathrm{~N}$ $(\nabla)$ and $f_{1, \max }=4.1 \mathrm{~N}(\bigcirc)$ with same values of $F_{0}=8 \mathrm{~N}$ and $F_{\perp}=0.15 \mathrm{~N} /$ grain. The amplitude decreases linearly along the chain and the slope of the decrease does not depend on $f_{1, \max }$.

between the sphere centroids is larger than the sum of the sphere radii.

For spheres in contact, the contact area is obviously a disk. Let us now consider equal cylinders of length $L$ and radii $R$. In the following we present the variations of the surface area, displacement and wave velocity for cylinders. Then we propose a simple model accounting for the pulse damping.

\section{Surface area}

In the case of two long cylinders (i.e. $L>>R$ ) in contact along a common generatrix, the contact area is a rectangle. The surface area $S$ varies with the compressive force as [24]:

$$
S=\sqrt{\frac{16 L R}{E^{*}} F}
$$

with $E^{*}=\pi E /\left(1-\nu^{2}\right)$.

\section{Displacement}

The theoretical relationship between $F$ and $\delta, F=$ $g(\delta)$, is more complex, and is given approximately, for the displacement (see [24]) by:

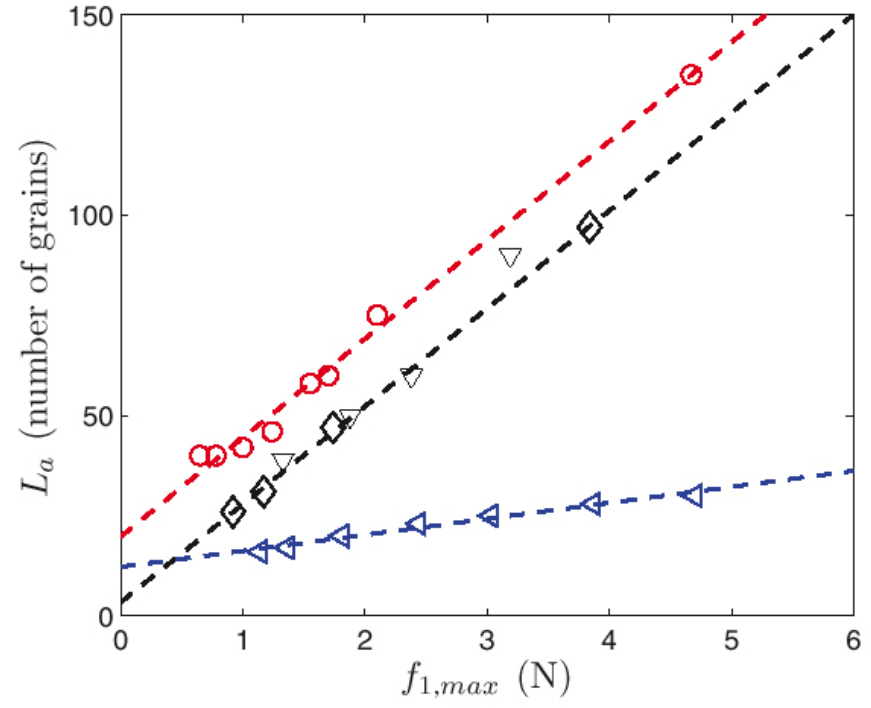

FIG. 12: (Color online) Distance of attenuation of the pulse given by Eq. (13) for different configurations: $(\diamond) F_{0}=8 \mathrm{~N}$, $F_{\perp}=0.15 \mathrm{~N} /$ grain, $(\nabla) F_{0}=14 \mathrm{~N}, F_{\perp}=0.15 \mathrm{~N} /$ grain, $(\bigcirc)$ $F_{0}=26 \mathrm{~N}, F_{\perp}=0.22 \mathrm{~N} /$ grain, $(\triangleleft) F_{0}=26 \mathrm{~N}$, and $F_{\perp}=$ $0.49 \mathrm{~N} /$ grain. As predicted by Eq. (13), $L_{a}$ increases linearly with $f_{1, \max }$. The slope is $1 / \alpha$. It seems to not depend on $F_{0}$ but decreases when $F_{\perp}$ increases.

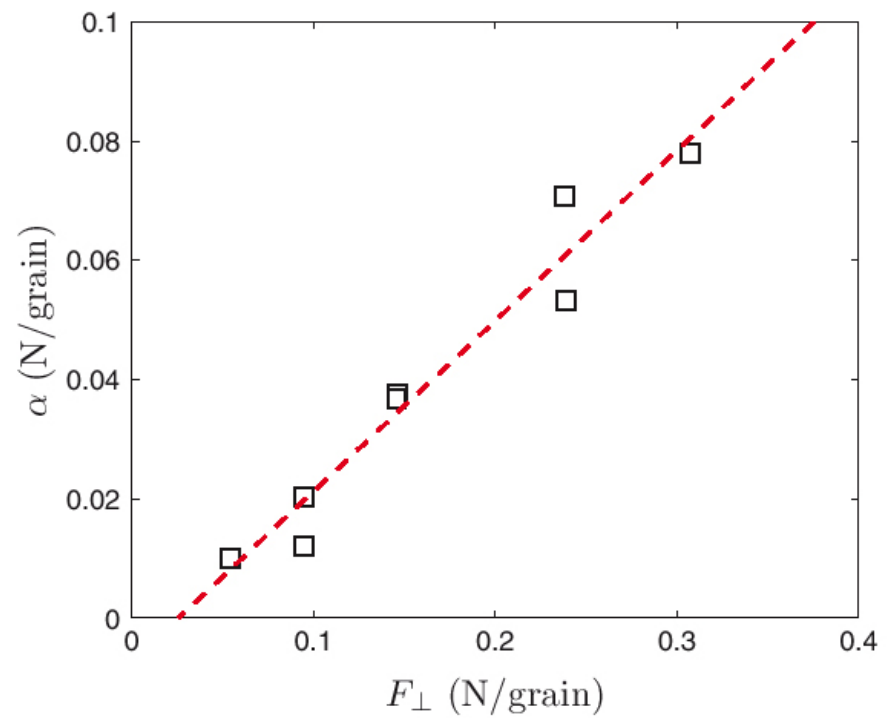

FIG. 13: (Color online) Plot of $\alpha$ as a function of $F_{\perp}$. We deduce from these experiments that: $\alpha=\mu F_{\perp}$, which is ana$\log$ to the Coulomb law of solid friction. The fit (dashed line) gives $\mu=0.28$.

$$
\delta=\frac{2 F}{L E^{*}}\left[\ln \left(\frac{4 R L E^{*}}{F}\right)-1\right]=g^{-1}(F)
$$

Of course, this law holds for ideal cylinders. 


\section{Wave velocity}

Thus, the system of cylinders maintained into contact by a static compressive load can be viewed as chain of masses $M=\rho \pi R^{2} L$ and nonlinear springs obeying Eq. 7. We note $u_{n}$ the displacement of the mass $n$ compared to its equilibrium position. The dynamics of this system is then described by the set of equations:

$$
M \ddot{u}_{n}=g\left(\delta_{0}-u_{n}+u_{n-1}\right)-g\left(\delta_{0}-u_{n+1}+u_{n}\right)
$$

$\delta_{0}$ is the displacement of the centroid due to the static force $F_{0}$ and depends on $F_{0}$ according to Eq. 7 (with $\delta=\delta_{0}$ and $F=F_{0}$ ).

In the linear regime $\left|u_{n}-u_{n-1}\right| \ll \delta_{0}$, the system (Eq. 8) can be linearized and reads therefore as:

$$
M \ddot{u}_{n}=k\left(u_{n+1}+u_{n-1}-2 u_{n}\right)
$$

where $k$ represent the stiffness of each contact:

$$
k^{-1}\left(F_{0}\right)=\frac{2}{L E^{*}}\left[\ln \left(\frac{4 R L E^{*}}{F_{0}}\right)-2\right] .
$$

The wave equation is linear in this regime but leads to a wave velocity $V$ which depends nonlinearly on the confining force and which reads as:

$$
V=2 R \sqrt{\frac{k\left(F_{0}\right)}{M}}
$$

Note moreover that both the Hertz law and Eq. 7 proceed from the equilibrium solutions of the elasticity equations. Therefore, the analogy with mass and spring systems (as exemplified in Eq. 8 or in Ref. [1]) relies implicitly on the hypothesis that the sound celerity through the material constituting the spheres or the cylinders is very large compared to the pulse velocity through the array.

\section{Dissipation}

The linear decay shown in Fig. 11 is consistent with the following equation:

$$
f_{n, \max }=-\alpha_{\left(F_{0}, F_{\perp}\right)}(n-1)+f_{1, \max }
$$

We attribute this linear decay of the pulse amplitude with distance to the solid friction with the cell walls.

From Eq. 12, we deduce the attenuation length $L_{a}$ (expressed in grain diameter unit):

$$
L_{a}=\frac{f_{1, \max }}{\alpha_{\left(F_{0}, F_{\perp}\right)}}
$$

On the other hand, it can be deduced from our measurements (see Fig. 13) that

$$
\alpha=\mu F_{\perp} .
$$

This relation corresponds to the law of Coulomb for the dry friction. A linear fit gives $\mu=0.28$. This value compares well to the measured coefficient of friction obtained from the onset of sliding of a grain deposited on an inclined plane made of the confining cell material. Thus, we conclude that the main process of dissipation originates in the friction between the cylinders and the slot frame. Such a dissipation mechanism has been highlighted for high amplitude wave propagation in a $1 \mathrm{D}$ bead chain by Job et al [25]. In that case the normal force was constant and only due to the grain weight.

Nevertheless, it is worth noting that the attenuation length $L_{a}$ does not exactly tends to zero as the pulse amplitude $f_{1, \max }$ tends to zero (see Fig. 12). It means that there are another sources of damping acting in parallel, but less efficient than the dry friction. This second source of dissipation likely originates from the polymer viscoelestic properties.

\section{B. Discussion}

The experimental data displayed in Fig. 6 show that the wave velocity $V$ exhibits two different regimes as the static confining force $F_{0}$ is varied. These two different regime can be fitted with a power law: $V \propto F_{0}^{\beta}$, with

$$
\beta \approx\left\{\begin{array}{lll}
1 / 4 & \text { if } \quad F_{0}<F_{c} \\
1 / 9 & \text { if } \quad F_{0}>F_{c}
\end{array}\right.
$$

The cross-over between these two regimes is found for $F_{c} \approx 20 \mathrm{~N}$. In the case of smoother surfaces, the exponent above the critical force is close to $1 / 11$ instead of $1 / 9$, which is closer to the expected theory for ideal cylinders.

For $F_{0}>F_{c}$, the experimental result closely meets the prediction expected from the spring-mass model, with a stiffness consistent with the logarithmic contact law suited for cylinders (Eq. 10).

On the other hand, for $F_{0}<F_{c}$, the dependence of the velocity with the static force is significantly larger than expected from Eq. 7. It results from the fact that, due to micro- and macroscale surface defects, the effective area of contact varies more strongly than expected with the compressive force. Interestingly, we notice a value for the exponent $\beta \approx 1 / 4$ which compares well with that reported for $1 \mathrm{D}, 2 \mathrm{D}$ and $3 \mathrm{D}$ packings of spheres ([4], [13], $[20],[21])$. The exponent seems to be non-significantly sensitive to the packing dimensionality and to the shape of bodies in contact. 
In the case of spheres packings, several hypotheses have been advanced to explain the deviation to the $V \propto F^{1 / 6}$ behavior expected from the Hertz law. Nevertheless, for the linear array studied here, the possibility of contact recruitment (which is a $2 \mathrm{D}$ or $3 \mathrm{D}$ effect) [26] must certainly be discarded. On the other hand, the hypothesis raised by Goddard [23] which involves the possible role played by the microscopical surface roughness must certainly be retained. For sufficiently low static force, the contact stiffness is prominently governed by the deformation of asperities, and, e.g., conical asperities would precisely lead to an exponent $\beta=1 / 4$.

In order to investigate the origin of the exponent $\beta$ in the law $V \propto F_{0}^{\beta}$, we have conducted measurements with two families of cylinders, with two different qualities of surface polishing. These measurements led to close results, except at high forces where an exponent $\beta=1 / 9$ is found for rough surfaces, and $\beta=1 / 11$ for smoother surfaces. The last value corresponds very closely to that expected for an array of ideal cylinders.

As shown in Fig. 9, the area of contact between two neighboring cylinders (shown as bright areas in the picture), is not always a rectangle, depending on the quality of the grain surfaces. A explanation for this feature can be that ylinders are compressed along their revolution axis while machined - so that, once freed of the machine, they dilate along the symmetry axis and, in turn, the radius at mid-height undergoes a contraction. This mechanism would thus implies a varying radius along the revolution axis. It might also be envisaged that the axes of the cylinders in contact are not perfectly parallel, but images of the contacts show that this is not the case. We observe that when the confining force $F_{0}$ is increased up to $F_{c} \approx 20 \mathrm{~N}$, the contact areas become closer to rectangles and that, in parallel, the theoretical behavior expected for a perfectly- aligned array of ideal cylinders is recovered. Our main conclusion is that imperfection in the perfect cylinder contact, both at micro- and macroscales leads to an exponent $\beta=1 / 4$ at low forces, and the ideal cylinder case is recovered for high forces.

\section{CONCLUSION}

We have studied the elastic wave propagation in a 1Dchain of polymeric cylinders. By means of photoelasticity and ultra-high speed visualization, we succeeded in hav- ing a real time access to the local state of stress of individual grains during the passing of an acoustic pulse. We have measured in parallel the variation in the geometry of the contact between neighboring grains as the static confining force $F_{0}$ is varied. In the linear regime of propagation, and for pulse widths of the order of six or seven grain sizes, we determined that the pulse velocity varies approximatively as a power law $V \propto F_{0}^{\beta}$ (with $\beta=1 / 4$ ) of the static confining force $F_{0}$, provided that the static force $F_{0}$ is smaller than $F_{c} \approx 20 \mathrm{~N}$. Surprisingly, the exponent $\beta=1 / 4$ compares with that found for packings of spherical grains, and deviates from the theoretical prediction suited for perfect arrays of equal cylinders. Experiments conducted with polished cylinders, or with cylinders of various roughness, lead to small changes at intermediate forces.

In the limit of the large confining forces $\left(F_{0}>>F_{c}\right)$, the relation between the pulse velocity and the confining force meets the theoretical prediction. A close look at the contact scale shows that the rectangular geometry of the contact, predicted for perfect cylinders, is recovered in this limit. On the other hand, the geometry of the contact area can deviates significantly from a rectangle for small confining forces.

The effects of a double power law for the contact on nonlinear waves propagation have been studied in [28] and [29]. In these references, the two behaviors come from the special geometry of the objects placed in the chain. In our study we also have a double power law, but due to a multiscale roughness. Another difference is the fact that the nonlinearity decreases with the force.

We have also studied the damping of the compressive pulse as it propagates through the array. We have first shown that the maximum amplitude $f_{n, \max }$ decreases linearly with the covered distance. We have also shown that the damping distance is a linear function of the normal load. These two features prove that the main source of dissipation originates in the solid friction with the cell walls. The experimental value of the friction coefficient corresponding to the wave damping matches well with that determined by measuring the onset of sliding of a grain deposited on an inclined wall surface.

\section{Acknowledgments}

We would like to thank the referees for their fruitful comments and suggestions on this paper.
[1] V.F. Nesterenko, J. Appl. Mech. Tech. Phys. 24, pp. 567575 (1983)

[2] C.H. Liu and S.R. Nagel, Phys. Rev. Lett. 68, pp. 23012304 (1992)

[3] C. Coste, E. Falcon and S. Fauve, Phys. Rev. E 56, pp. 6104- 6117 (1997).
[4] X. Jia, C. Caroli and B. Velicky, Phys. Rev. Lett., 82, pp. 1863-1866 (1999)

[5] X. Jia, Phys. Rev. Lett. 93, pp. 154303 (2004)

[6] V. Tournat, V.E. Gusev, and B. Castagnède, Phys. Rev. E 66, pp. 041303 (2002)

[7] V. Tournat, V. Gusev, B. Castagnede. Physics Letters A 
326,pp. 340-348 (2004)

[8] L.D. Landau, E.M. Lifshitz, Theory of Elasticity (Pergamon Press, Oxford, 1986)

[9] H.J. Hermann, D. Stauffer and S. Roux, Europhysics Lett. 3, pp. 265-267 (1987)

[10] F. Radjai, M. Jean, J.J. Moreau, et al., Phys. Rev. Lett. 77, pp. 274-277 (1996)

[11] V.F. Nesterenko, Dynamics of Heterogeneous Materials, Springer, New York (2001)

[12] C. Coste and B. Gilles, Eur. Phys. J. B 7, pp. 155-168 (1999).

[13] B. Gilles, C. Coste, Phys. rev. Lett. 90174302 (2003)

[14] C. Daraio, V.F. Nesterenko, E.B. Herbold, et al., Phys. Rev. E 72, 016603 (2005)

[15] S. Job, F. Melo, A. Sokolow, S. Sen, Granular Matter 10, pp. 13-20 (2007)

[16] A. Shukla, Optics and Lasers in Engineering 14, pp. 165184 (1991)

[17] A. Shukla, M. H. Sadd, R. Singh, Q. M. Tai and S. Vishwanathan, Optics and Lasers in Engineering 19, pp. 99119 (1993)

[18] M. H. Sadd, Q. M. Tai, A. Shukla, Int. Journal of NonLinear Mechanics, 28, 2, pp. 251-265 (1993)

[19] X. Noblin, G. Huillard, and J. Rajchenbach, 6th Inter- national Conference on the Micromechanics of Granular Media, July 13-17, 2009 Golden, CO. Powders and Grains 2009 AIP Conference Proceedings. 1145, p. 439 (2009).

[20] M. de Billy, Journal of the Acoustical Society of America, 108, 4, pp. 1486- 1495 (2000)

[21] J. Duffy and R.D. Mindlin, J. Appl. Mech. 24, pp. 585593 (1957)

[22] J.N. Roux, Phys. Rev. E 61, pp. 6802-6836 (2000).

[23] J.D. Goddard, Proc. Roy. Soc. (London) A 430, pp. 105131 (1990)

[24] K.L. Johnson, Contact mechanics, Cambridge University Press (1992)

[25] Job S, Melo F, Sokolow A, et al., Phys. Rev. Lett. 94, $178002(2005)$

[26] T. Travers, M. Ammi, D. Bideau, A. Gervois, J.-C. Messager, J.-P. Troadec J. Phys. France 49, 939 (1988)

[27] P.A. Johnson and X. Jia, Nature 437, pp. 871-874 (2005)

[28] E.B. Herbold, V.F. Nesterenko, Appl. Phys. Lett., 90, pp. 261902 (2007)

[29] I.L.D. Pinto, A. Rosas, A.H Romero, et al, Phys. Rev. E 82, pp. 031308 (2010) 